\title{
A ULTRASSONOGRAFIA PULMONAR COMO MÉTODO PREDITOR DE SUCESSO OU FALHA DE DESMAME DA VENTILAÇÃO MECÂNICA
}

\section{ARTIGO DE REVISÃO}

MELO, Jefferson José Silva de ${ }^{1}$

MELO, Jefferson José Silva de. A ultrassonografia pulmonar como método preditor de sucesso ou falha de desmame da ventilação mecânica. Revista Científica Multidisciplinar Núcleo do Conhecimento. Ano. 07, Ed. 01, Vol. 06, pp. 0515. Janeiro de 2022. ISSN: 2448-0959, Link de acesso: https://www.nucleodoconhecimento.com.br/saude/ultrassonografia-pulmonar

\section{RESUMO}

A avaliação dos pulmões, realizada através da Ultrassonografia Pulmonar (USP) é um assunto de grande relevância para a comunidade científica no que tange ao exame de pacientes críticos submetidos a Suporte Ventilatório Invasivo. Este recurso é baseado no fato de que as complicações pulmonares diminuem a aeração no interior dos pulmões. Tal método pode fornecer parâmetros complementares ao exame físico e clínico, tendo a vantagem de ser realizado à beira do leito, além de permitir firmar diagnósticos e monitorar intervenções terapêuticas. O objetivo deste artigo científico foi realizar uma revisão de literatura, por meio de pesquisa bibliográfica, e responder à seguinte Pergunta Norteadora: a Ultrassonografia Pulmonar é um método preditor de sucesso ou falha do processo de desmame da Ventilação Mecânica na Unidade

1 Pós-Graduado em Fisioterapia Cardiorrespiratória e Fisioterapia em Terapia Intensiva Adulto, Centro Universitário Augusto Motta - UNISUAM, Rio de Janeiro/RJ, graduado em Fisioterapia pela Universidade Estácio de Sá-UNESA. ORCID: 00000003-3660-339

RC: 105610

Disponível em: https://www.nucleodoconhecimento.com.br/saude/ultrassonografia-pulmonar 
de Terapia Intensiva? Para o desenvolvimento da pesquisa, entre os dias 01 setembro e 04 outubro de 2021 foi realizada uma busca sistemática na literatura científica para seleção de artigos referentes à temática em estudo, publicados no período de 20042019, utilizando Google Acadêmico, PubMed, Lilacs, Medline, Scielo, PEDro. Dos 20 artigos localizados, 14 foram selecionados para a leitura na íntegra, dentre os quais 08 foram excluídos: 04 por não descreverem o uso da USP e 04 por não corresponderem ao período cronológico delimitado. Ao final, foram escolhidos 06 artigos (dentre eles: estudo longitudinal, estudo exploratório, estudo comparativo transversal, ensaio clínico, série de caso e estudo de viabilidade randomizado), através dos quais foi possível inferir que a Ultrassonografia é um método de diagnóstico muito utilizado na Unidade de Terapia Intensiva (UTI) devido ao seu baixo custo, por não ser uma conduta invasiva, de rápida compreensão e por proporcionar todas as informações sobre diferentes tecidos e órgãos, sendo útil também na avaliação da disfunção e atrofia do diafragma em pacientes graves e em Ventilação Mecânica (VM). Com o resultado apontado, evidenciou-se que este exame é bem estabelecido na literatura, tendo, em seu uso, o objetivo de obter sucesso no processo de desmame e extubação da VM, pois demonstra resultados preditores.

Palavras-chave: Ultrassonografia Pulmonar, Extubação, Desmame, Ventilação Mecânica.

\section{INTRODUÇÃO}

O número de ocorrências de complicações pulmonares referentes a ventilação mecânica é uma questão considerável na atenção aos pacientes graves. A diminuição do tempo da assistência respiratória é fundamental para reduzir complicações. A extubação de um doente caracteriza o fim do processo de desmame. Infelizmente, ainda depois de tentar uma Respiração Espontânea (Teste de Respiração Espontânea - TRE) com sucesso, aproximadamente 30\% dos pacientes apresentam desconforto respiratório durante as primeiras 48 horas pós-extubação em decorrência 
de insucesso do procedimento, demandando ventilação mecânica não invasiva ou até mesmo reintubação (BOLES et al., 2007).

A redução da aeração no interior dos pulmões depois do processo de retirada do tubo endotraqueal (extubação) é caracterizante de falha do procedimento, levando ao déficit da troca gasosa, aumentando a duração da ventilação artificial e provocando acréscimo nas taxas de morbidade e mortalidade (PEÑEULAS; FERNÁNDEZ; ANZUETO, 2011).

A Ultrassonografia é um recurso eficaz para prever sucesso ou falha do processo extubação, sendo cada vez mais conhecido e empregado na Unidade de Terapia Intensiva (UTI) por profissionais fisioterapeutas, por ser capaz de identificar o deslocamento diafragmático durante o ciclo respiratório espontâneo ou assistido, mostrando a eficácia do diafragma de promover força e, consequentemente, volume corrente. O número de estudos acerca dessa aplicação na avaliação diafragmática tem crescido a cada ano.

Este artigo científico objetiva discorrer sobre o seguinte questionamento: a Ultrassonografia Pulmonar é um método eficaz para prever sucesso ou falha do processo de desmame da Ventilação Mecânica na Unidade de Terapia Intensiva?

\section{DESENVOLVIMENTO}

\subsection{MECANISMOS DE GERAÇÃO DA IMAGEM DA USP}

As características normais encontradas na USP se definem como imagem brilhante e clara, ou seja, hiperecoica, revestida pela pleura, em função da interface refletidora (pulmão preenchido de ar), bloqueando a propagação do feixe de ultrassom. À proporção que o pulmão concentra líquido na zona intersticial, a representação vai se tornando cada vez mais visível (SOLDATI et al., 2008).

RC: 105610

Disponível em: https://www.nucleodoconhecimento.com.br/saude/ultrassonografia-pulmonar 
Existe um postulado ecográfico que afirma que o ultrassom é refletido através de uma interface entre desiguais impedâncias acústicas, logo, a imagem do pulmão saudável é representada por elevada impedância, sem acustic mismatch (que em tradução livre significa "pausa acústica") na trajetória interna da cavidade torácica (PICANO, 2006; VOLPICELLI, 2008; JAMBRICK, 2004).

O espaço pleural é situado a $01 \mathrm{~cm}$ internamente à parede torácica. $\mathrm{O}$ pulmão cheio de ar, revestido por uma película denominada pleura visceral, é um vigoroso transmissor do feixe de ultrassom, se apresentando como uma clara e brilhante interface de forma linear e movendo-se de acordo com os movimentos respiratórios (sinal do deslizamento), tornando-se um marcador do revestimento dos pulmões (a pleura visceral) e demonstrando sua localização. A pleura parietal se apresenta como uma linha de menor distinção, com pequeno foco ecogênico, determinadas vezes ofuscada por algum objeto de reflexão, conhecida como Localização Inferida (RUMACK et al., 2006).

O ar é o principal componente encontrado no interior pulmonar, sua impedância acústica é bastante diferente de um osso, do parênquima pulmonar e, também, da água. Levando em consideração que um pulmão sadio contém pouco líquido e muito ar em seu interior, as Linhas B (figura 2) são identificadas como uma ínfima estrutura farta em água, sendo contornada por ar, originando um elevado gradiente de impedância. Nos pulmões de pessoas normais, ou seja, indivíduos saudáveis, não são percebidos tecidos intersticiais, inclusive por tomografia computadorizada torácica (TC-T) (LICHTENSTEIN et al., 2005).

Nos apanhados ecográficos, é relevante distinguir as Linhas A (figura 1) das Linhas B (figura 2). As primeiras são linhas horizontais que representam reflexões acústicas, normalmente encontradas em pulmões sadios, não caracterizando modificações patológicas. Linhas A são verificadas como artefatos ligeiramente ecogênicos entre as regiões sombrias das costelas, uma vez que o transdutor está na posição longitudinal (VOLPICELLI et al., 2008). 
Figura 1 - Superfície normal do pulmão.

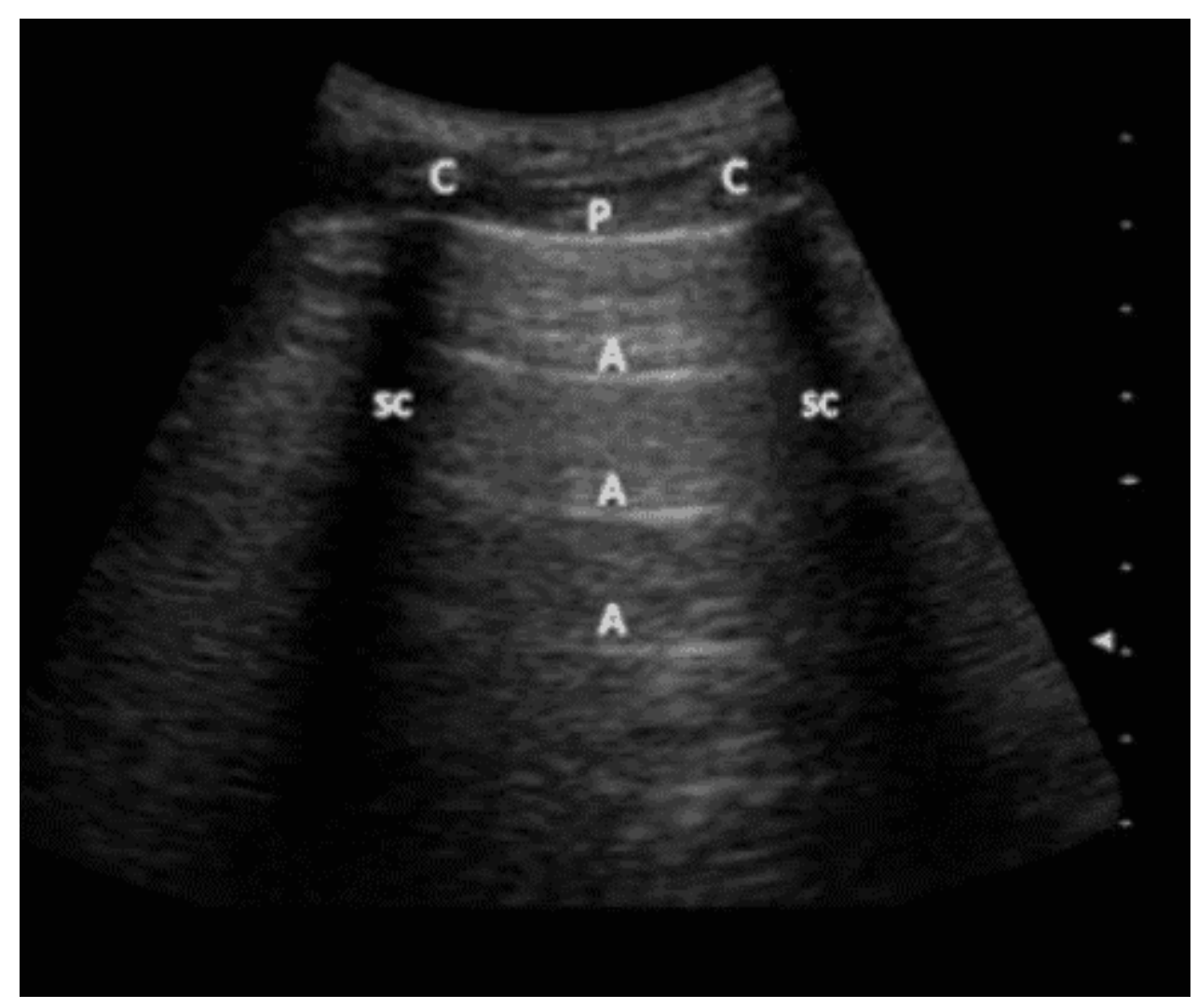

Fonte: Faistauer et al., 2010.

$\mathrm{Na}$ figura 1 é perceptível o scan/scanner longitudinalmente a um espaço intercostal. A linha pleural $(P)$, em um indivíduo adulto, está localizada a $1 / 2 \mathrm{~cm}$ inferiormente à linha costal (C), apresentando dimensão de aproximadamente 2 centímetros. A imagem mostra também as linhas da sombra acústica costal (SC), além das linhas A, paralelamente horizontais à linha pleural e descontinuadas entre si por distâncias regulares, semelhantes à da pele, até a linha pleural. 
Figura 2 - Superfície anormal do pulmão.

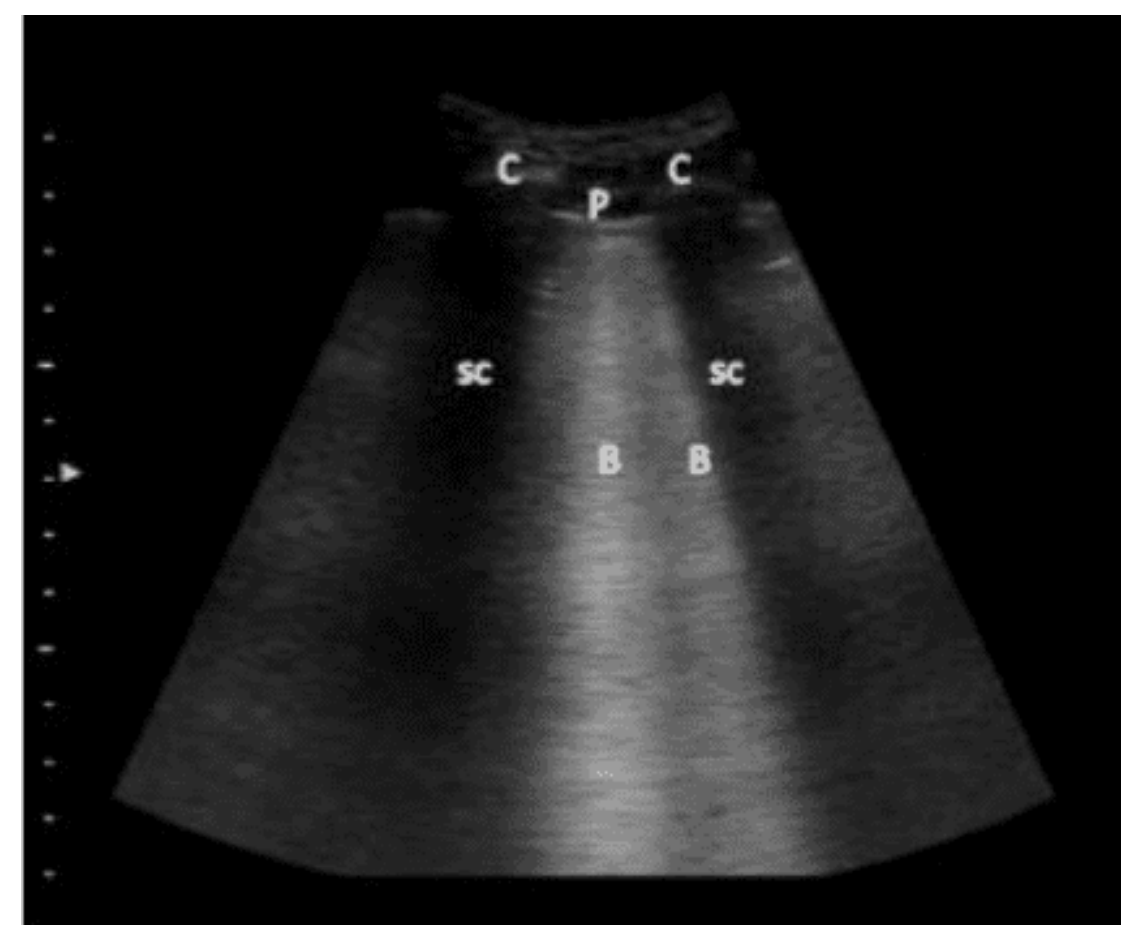

Fonte: Faistauer et al., 2010.

De acordo com Faistauer et al. (2010), a figura 2 evidencia uma USP contendo duas Linhas B, denominadas Sinais da Cauda de Cometa (SCC). Estes sinais são verticais, hiper-ecogênicos, apresentando algumas características: (1) originam-se na linha pleural; (2) são bastante delineados; (3) difundidos a partir da linha pleural e contínuos até a extremidade oposta, sem interrupção do sinal; (4) acompanham os movimentos da pleura e do pulmão; e (5) anulam as linhas A. A imagem evidencia ainda as costelas $(C)$, a linha pleural $(P)$, a sombra acústica costal (S) e as linhas $B$.

\subsection{EVIDÊNCIAS NA UTILIZAÇÃO DA ULTRASSONOGRAFIA PULMONAR NO CONTEXTO CLÍNICO}

Muitos estudos têm comprovado que é de grande utilidade o uso da USP em pacientes com queixas respiratórias, nos setores de emergência e de terapia intensiva. Em um deles, Lichtenstein et al. (2004) observaram o uso desta técnica em pacientes com 
Síndrome da Angústia Respiratória Aguda (SARA) em comparação ao uso de Radiografia Torácica (RX-Tórax), de Ausculta Pulmonar e de Tomografia Computadorizada de Tórax no diagnóstico e contenção do progresso da enfermidade, buscando identificar o método mais eficiente para análise da precisão diagnóstica. No referido trabalho, foram avaliados 32 doentes com SARA e 10 indivíduos saudáveis (voluntários), sendo evidenciada a superioridade de eficiência da USP em relação aos demais métodos para diagnosticar Derrame Pleural, Consolidação (endurecimento) Pulmonar e Síndrome Alvéolo-Intersticial (SAI), além de possibilitar a mensuração das dimensões da lesão. É possível que a SARA se apresente juntamente com a mencionada síndrome e ainda com diversos achados pulmonares simultaneamente, tais como: Consolidação Pulmonar e Derrame Pleural. Esses distúrbios podem ser detectados pela ultrassonografia, que é de fácil realização em pacientes restritos ao leito e em ventilação artificial, estado no qual se mantêm os doentes com SARA. O supracitado autor defende a utilização desse método para controle de focos de infecção nas Unidades de Terapia Intensiva (UTI), por seu acesso ao leito ser mais fácil.

A Ultrassonografia Pulmonar permite ainda uma avaliação padronizada de pacientes com dispneia e/ou insuficiência respiratória (MAYO, 2009; ANANTHAM, 2010), baseada no perfil dos achados da USP em conjunto com a avaliação para trombose venosa nos membros inferiores.

Este exame também pode ser um método útil na avaliação da função diafragmática, através da análise da sua movimentação na inspiração profunda, do volume corrente e do sniff test (KOENIG, et al. 2011).

Além disso, a movimentação diafragmática pode ser um bom fator preditor para o sucesso da extubação de pacientes críticos, enquanto a drenagem torácica guiada pelo ultrassom pode acelerar o desmame da ventilação mecânica (HAYAT; KHAN; ASGHAR, 2017). 


\subsection{DESMAME}

O processo de desmame é compreendido pela gradual remoção do suporte ventilatório invasivo à retirada total do tubo endotraqueal e pode corresponder a aproximadamente 42\% do tempo da Ventilação Mecânica - VM (TOBIN, 2012; BOLES, 2007).

A aplicação da Tentativa de Respiração Espontânea - TRE tem suplantado a utilização de inúmeros sinais respiratórios para estabelecer o processo de desmame na UTI, por conta de sua utilização mais rápida e prognosticadora (MACINTYRE, 2012).

A aplicação de melhores avaliações do doente antes e depois da TRE é de extrema importância para a previsão do insucesso no processo de desmame e para evidenciar um tratamento que possibilite a redução do período de utilização do suporte ventilatório artificial. As razões para falha do desmame do suporte com VM são frequentemente multifatoriais e envolvem uma complexa inter-relação entre disfunções cardíacas e pulmonares. Uma recente revisão sugere que o intensivista pode usar a USP de forma produtiva para identificar impedimentos a uma extubação bem-sucedida (MAYO et al., 2016).

\section{MATERIAIS E MÉTODOS}

Para o desenvolvimento desta pesquisa, foi realizada, entre os dias 01 setembro e 04 outubro de 2021, uma revisão sistemática na literatura científica de artigos referentes a esta temática publicados no período de 2004-2019, através de busca eletrônica nas bases de dados PubMed, Lilacs, Medline, Scielo, PEDro, Google Acadêmico e edições recentes de livros, utilizando combinações dos descritores "ultrassonografia", "extubação", "desmame" e "ventilação mecânica", bem como termos equivalentes em inglês. Os critérios de inclusão para este estudo foram ensaios clínicos controlados e randomizados, estudos retrospectivos, publicados no período de 2004 a 2019, nos idiomas inglês e português, que abordassem o uso de USP na previsão de falha de desmame de ventilação mecânica. Foram enquadrados nos critérios de exclusão, os RC: 105610 
artigos que não versavam sobre o uso da USP e os que não obedeciam ao período cronológico de publicação. Dos 20 artigos averiguados, 14 foram selecionados para a leitura na íntegra, sendo que destes 04 foram excluídos por não descreverem o uso da USP e 04 por não corresponderem ao período cronológico estabelecido. Ao final, foram selecionados 06 artigos, incluindo: estudo longitudinal, estudo exploratório, estudo comparativo transversal, ensaio clínico, série de caso e estudo de viabilidade randomizado.

Figura 3 - Fluxograma

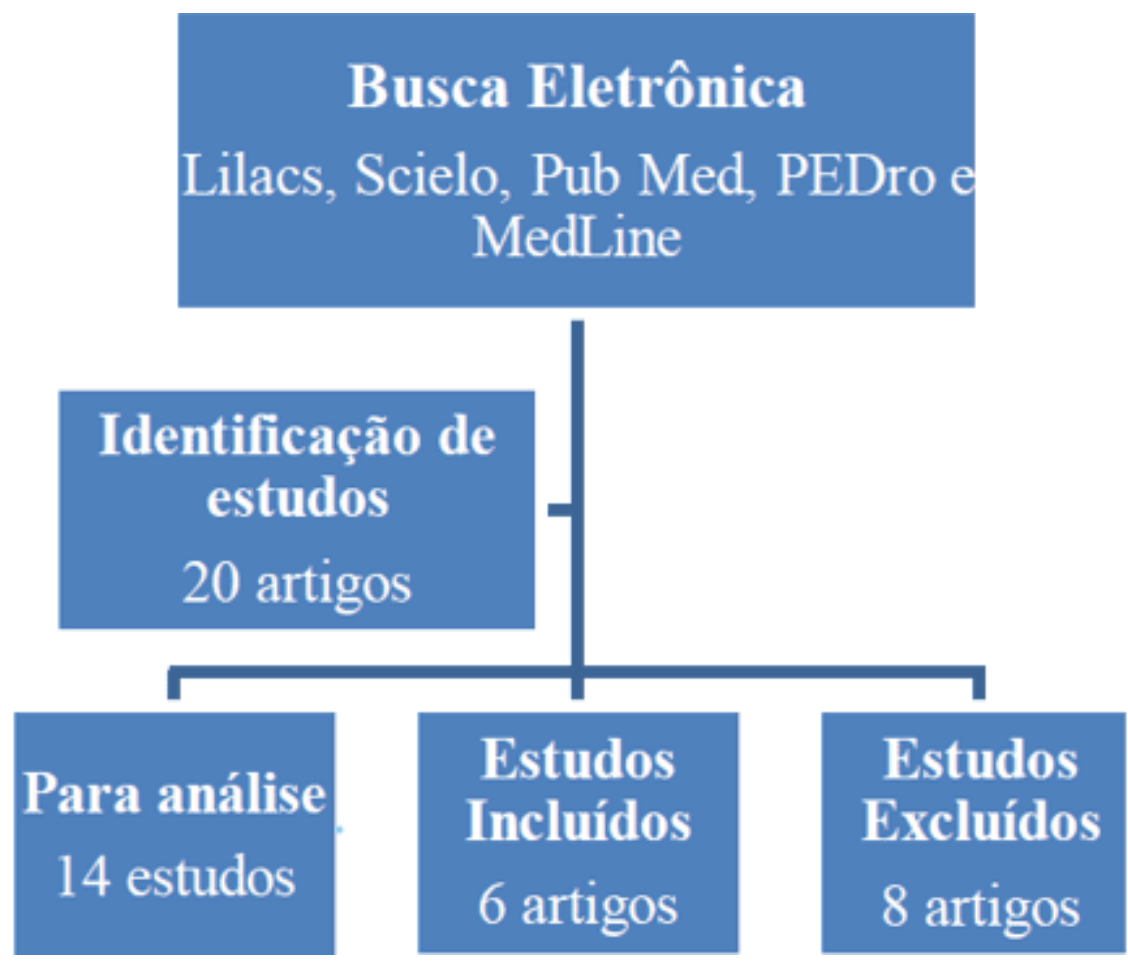

Fonte: Autor, 2021.

\section{RESULTADOS E DISCUSSÃO}

Foram encontrados vinte artigos e, após a leitura dos resumos, foram selecionados quatorze artigos para leitura na íntegra. A partir desta leitura, foram excluídos oito trabalhos, dentre os quais quatro não descreviam o uso da USP, dificultando, assim, a reprodutibilidade; e outros quatro não correspondiam ao período cronológico. Ao RC: 105610

Disponível em: https://www.nucleodoconhecimento.com.br/saude/ultrassonografia-pulmonar 
final das buscas e leitura dos artigos, foram selecionados seis, incluindo: estudo longitudinal, estudo exploratório, estudo comparativo transversal, ensaio clínico, série de caso e estudo de viabilidade randomizado.

Hayat et al. (2017). mensuraram, por ultrassom, a excursão diafragmática e seu resultado no desmame da ventilação mecânica. Os pacientes foram acompanhados por quarenta e oito horas e classificados de acordo com o desfecho como desmame bem-sucedido e falha no desmame. Dos cem casos, setenta e seis pacientes tiveram um desmame bem-sucedido, enquanto vinte e quatro tiveram um resultado de desmame com falha. Em uma excursão diafragmática de 1,2 cm e mais, dos sessenta e sete casos, sessenta tiveram desmame bem-sucedido $(89,55 \%)$, enquanto sete casos (10,45\%) apresentaram falha no desmame. Em uma excursão inferior a 1,2 cm, dezessete dos trinta e três casos $(51,5 \%)$ tiveram desmame bem-sucedido enquanto dezesseis (48,48\%) apresentaram falha no desmame. Nesse ponto de corte $(1,2 \mathrm{~cm})$, a sensibilidade e a especificidade para o desmame bem-sucedido foram $78,95 \%$ e $70,83 \%$, respectivamente.

Dinino et al. (2013) concluíram que, quando a fração de espessamento do diafragma (fórmula: espessura no final da inspiração menos espessura no final da expiração dividido pela espessura no final da expiração) é maior do que 30\%, há uma maior acurácia, especificidade e valor preditivo negativo em predizer sucesso de extubação, se comparado ao índice de Tobin.

Já um estudo de Kim et al. (2011) publicado na Critical Care Medicine, demonstrou que pacientes com incursão diafragmática menor que $10 \mathrm{~mm}$, apresentavam maior tempo em ventilação mecânica e maior taxa de falha no desmame.

Outro trabalho, realizado por Zambon (2016), avaliou diversas variáveis, inclusive as utilizadas no cotidiano, e demonstrou que apenas os valores ultrassonográficos tiveram relevância estatística em predizer sucesso de extubação (com exceção do índice $\mathrm{P} / \mathrm{F})$.

RC: 105610

Disponível em: https://www.nucleodoconhecimento.com.br/saude/ultrassonografia-pulmonar 
El Naggar et al. (2019), avaliaram a mobilidade diafragmática e a fração de espessamento diafragmático de quarenta pacientes. Todos os achados ultrassonográficos foram reunidos e comparados com algumas das ferramentas usuais de desmame, como gasometria arterial e mecânica respiratória. Foi observado que trinta e um pacientes revelaram liberação bem-sucedida da ventilação mecânica. A mobilidade diafragmática e a fração de espessamento apresentaram alta sensibilidade e especificidade em comparação com outras ferramentas de desmame. O valor de corte foi de $10 \mathrm{~mm}$ para mobilidade e $30 \%$ para a fração de espessamento diafragmático. Concluiu-se que a Ultrassonografia pode ser utilizada como uma nova ferramenta para predição do processo de desmame.

Para finalizar, uma metanálise da Intensive Care Medicine, com vinte estudos, concluiu que a ultrassonografia é válida para diagnosticar disfunção diafragmática e predizer falha de desmame (ZAMBON, 2016).

\section{CONSIDERAÇÕES FINAIS}

Com base nesta pesquisa, cujo objetivo foi obter resposta se a Ultrassonografia Pulmonar é um método eficiente para prever sucesso ou falha no processo de desmame de pacientes submetidos à Ventilação Artificial, foi possível concluir que a USP tem, cada dia mais, sua presença consolidada no exame do paciente crítico. Se ela for associada à rotina de avaliação dos pacientes em desmame ventilatório, possivelmente teremos menores taxas de falhas de extubação. Um exame focado e simples, dicotômico, que permite predizer sucesso ou falha de desmame de ventilação mecânica. Possui inúmeras vantagens como aprendizado fácil, método rápido, não invasivo e de fácil reprodução, equipamento portátil (a avaliação à beira do leito, em tempo real), dispensa a necessidade e risco do transporte do paciente, limita a exposição à radiação. Todavia, é extremamente necessário um acesso contínuo à essa ferramenta.

Este artigo evidenciou que a medida ultrassonográfica da excursão diafragmática é um bom método para prever o desmame da ventilação mecânica, uma vez que a RC: 105610

Disponível em: https://www.nucleodoconhecimento.com.br/saude/ultrassonografia-pulmonar 
percepção da diminuição da mobilidade e da espessura do diafragma por meio da ultrassonografia é comprovadamente um bom preditor de insucesso do desmame da VM. Concluindo-se, então, que a Ultrassonografia Pulmonar é um eficiente método preditor de sucesso ou falha no processo de desmame da Ventilação Mecânica na Unidade de Terapia Intensiva.

\section{REFERÊNCIAS}

ANANTHAM D., et al. Ultrasonography. Murray and Nadel's textbook of respiratory medicine. 5th ed. Philadelphia: Saunders-Elsevier; 2010. p. 445-60.

BOLES, J., et al. Weaning from mechanical ventilation. Eur Resp J. 2007; 29(5):1033-56.

DININO, E., et al. Diaphragm ultrasound as a predictor of successful extubation from mechanical ventilation. CRITICAL CARE. 2013.

EL NAGGAR, T. H., et al. Diaphragm ultrasound as a predictor of successful extubation from mechanical ventilation. Egypt J Bronchol 2019;13:191-5.

FAISTAUER, A., et al. Ultrassonografia pulmonar na insuficiência cardíaca agudamente descompensada. Scientia Medica (Porto Alegre) 2010; volume 20, número 2, p. 194-199.

HAYAT, A.; KHAN, A.; ASGHAR, A. Diaphragmatic excursion: Does it predict successful weaning from mechanical ventilation? J Coll Physicians Surg Pak 2017;27(12):743-6.

JAMBRIK, Z., et al. Extravascular lung water. Am J Cardiol. 2004; 93:1265-70.

KIM, W. Y., et al. Diaphragm dysfunction assessed by ultrasonography: influence on weaning from mechanical ventilation. Crit Care Med. 2011;39(12):2627-30. http://dx.doi.org/10.1097/ccm.0b013e3182266408. 
KOENIG, S. J., et al. Thoracic ultrasonography for the pulmonary specialist. Chest. 2011;140(5):1332-41. PMid:22045878. http://dx.doi.org/10.1378/chest.110348.

LICHTENSTEIN, D.; et al. Comparative diagnostic performances of auscultation, chest radiography, and lung ultrasonography in acute respiratory distress syndrome. Anesthesiology. 2004;100:9-15.

LICHTENSTEIN, D., et al. Ultrasound diagnosis of occult pneumothorax. Crit Care Med. 2005;33:1231-8.

MACINTYRE, N. R. Evidence-based assessments in the ventilator discontinuation process. Respir Care. 2012;57(10):1611-8.

MAYO, P.; VOLPICELLI, G.; LEROLLE, N.; SCHREIBER, A.; DOELKEN, P.; VIEILLARD-BARON, A. Ultrasonography evaluation during the weaning process: the heart, the diaphragm, the pleura and the lung. Intensive Care Med. 2016;42(7):1107-17.

MAYO, P. H. Ultrasound evaluation of the lung. In: LEVITOV, A.; MAYO, P. H.; SLONIM, A. D., editors. Critical care ultrasonography. New York: McGraw-Hill; 2009. p. 251-8.

PEÑUELAS, O.; FERNÁNDEZ, C.; ANZUETO, A. Ventila Group. Characteristics and outcomes of ventilated patients according to time to liberation from mechanical ventilation. Am J Resp Crit Care Med. 2011;184(4):430-7.

PICANO, E., et al. Ultrasound lung comets: a clinically useful sign of extravascular lung water. J Am Soc Echocardiogr. 2006;19:356-63.

RUMACK, C. M.; WILSON, S. R.; CHARBONEAU, J. W. Tratado de ultra-sonografia diagnóstica. $3^{\text {a }}$ ed. Rio de Janeiro: Mosby Elsevier; 2006. 
SOLDATI, G.; GARGANI, L.; SILVA, F. R. Acute heart failure: new diagnostic perspectives for the emergency physician. intern Emerg Med. 2008;3:37-41.

TOBIN, M. J.; JUBRAN, A. Weaning from mechanical ventilation. In: TOBIN, M. J., editor. Principles and practice of mechanical ventilation. 3rd ed. New York: McGrawHill; 2012. p. 1185-220.

VOLPICELLI, G.; CARAMELLO, V.; CARDINALE, L.; et al. Bedside ultrasound of the lung for the monitoring of acute decom- pensated heart failure. Am J Emerg Med. 2008;26:585-91.

VOLPICELLI, G.; MUSSA, A.; GAROFALO, G.; et al. Bedside lung ultrasound in the assessment of alveolar-interstitial syndrome. Am J Emerg Med. 2008;24:689-96.

ZAMBON, M. Assessment of diaphragmatic dysfunction in the critically ill patient with ultrasound: a systematic review. Intensive Care Medicine, 2016.

Enviado: Outubro, 2021.

Aprovado: Janeiro, 2022. 